\title{
SOME VARIATIONS OF PERFECT GRAPHS
}

\author{
Magda Dettlaff, Magdalena Lemańska \\ Faculty of Applied Physics and Mathematics \\ Gdańsk University of Technology \\ ul. Narutowicza 11/12, 80-233 Gdańsk, Poland \\ e-mail: mdettlaff,magda@pg.gda.pl \\ Gabriel SemanišIn \\ Institute of Computer Science \\ P.J. Šafárik University \\ Jesenná 5, 04154 Košice, Slovakia \\ e-mail: gabriel.semanisin@upjs.sk
}

AND

Rita Zuazua

Faculty of Science

National Autonomous University of Mexico

Av. Universidad 3000, Mexico City, Mexico

e-mail: ritazuazua@ciencias.unam.mx

\begin{abstract}
We consider $\left(\psi_{k}-\gamma_{k-1}\right)$-perfect graphs, i.e., graphs $G$ for which $\psi_{k}(H)=$ $\gamma_{k-1}(H)$ for any induced subgraph $H$ of $G$, where $\psi_{k}$ and $\gamma_{k-1}$ are the $k$-path vertex cover number and the distance $(k-1)$-domination number, respectively. We study $\left(\psi_{k}-\gamma_{k-1}\right)$-perfect paths, cycles and complete graphs for $k \geq 2$. Moreover, we provide a complete characterisation of $\left(\psi_{2}-\gamma_{1}\right)$ perfect graphs describing the set of its forbidden induced subgraphs and providing the explicit characterisation of the structure of graphs belonging to this family.
\end{abstract}

Keywords: $k$-path vertex cover, distance $k$-domination number, perfect graphs.

2010 Mathematics Subject Classification: 05C69. 
[1] C. Berge, Färbung von Graphen, deren samtliche bzw. deren ungerade Kreise starr sind, Wiss. Z. Martin-Luther-Univ. Halle-Wittenberg Math.-Natur. Reihe 10 (1961) 114.

[2] A. Brandstädt, V.B. Le and J.P. Spinrad, Graph Classes: A Survey (Monographs on Discrete Math. Appl.) (SIAM, Philadelphia, 1999). doi:10.1137/1.9780898719796

[3] B. Brešar, F. Kardoš, J. Katrenič and G. Semanišin, Minimum k-path vertex cover, Discrete Appl. Math. 159 (2011) 1189-1195.

doi:10.1016/j.dam.2011.04.008

[4] G.S. Domke, J.H. Hattingh and L.R. Markus, On weakly connected domination in graphs II, Discrete Math. 305 (2005) 112-122. doi:10.1016/j.disc.2005.10.006

[5] T. Haynes, S. Hedetniemi and P. Slater, Fundamentals of Domination in Graphs (Marcel Dekker, 1998).

[6] M.A. Henning, O.R. Oellermann and H.C. Swart, Bounds on distance domination parameters, J. Combin. Inform. System Sci. 16 (1991) 11-18.

[7] M.A. Henning, O.R. Oellermann and H.C. Swart, Relationships between distance domination parameters, Math. Pannon. 5 (1994) 69-79.

[8] L. Volkmann, On graphs with equal domination and covering numbers, Discrete Appl. Math. 51 (1994) 211-217. doi:10.1016/0166-218X(94)90110-4

[9] I.E. Zverovich, Perfect-connected-dominant graphs, Discuss. Math. Graph Theory 23 (2003) 159-162. doi:10.7151/dmgt.1192

Received 3 March 2015

Revised 23 September 2015

Accepted 9 October 2015 